\title{
A Moving Target Tracking Method Based on Improved Meanshift Algorithm
}

\author{
Yue Zhou ${ }^{1,2, *}$, Janwei $\mathrm{Ji}^{1}$ and Kai Song ${ }^{1,2}$ \\ ${ }^{I}$ College of Information and Electrical Engineering, Shenyang Agricultural University, Shenyang, 110866, China; \\ ${ }^{2}$ School of Information Science \& Engineering, Shenyang Ligong University, Shenyang, 110159, China
}

\begin{abstract}
In this paper, an improved method are put forward for making up the defects of algorithm Meanshift in Moving target tracking, the automatic initialization of moving targets is realized by determining the center of mass, pixel location is determined by calculation the center of the target in each frame. The experimental results show that the improved Meanshift algorithm avoids the error of the artificial participation, reduces the tracking deviation phenomena and the number of iterations when calculating the candidate target center. The tracking accuracy and efficiency are improved.
\end{abstract}

Keyword: Automatic initialization, meanshift, moving target tracking, pixel location.

\section{INTRODUCTION}

Meanshift algorithm [1] is a kind of widely used moving target tracking algorithm. The target model was based on the color of the moving target feature modeling, Constant iterative search feature looking for maximum similarity of candidate targets followed the direction of the sample probability density increases. This algorithm have the advantage of limited number of iterations and less amount of calculation with a strong target tracking in real time [2]. Meanshift algorithm also has to be initialized manually, unable to locate the color distribution [3], etc. In this paper, We analyze the shortcomings of the traditional algorithmthe and put forward the improvement methods, the accuracy of target tracking was improved.

\section{THE PROBLEMS EXISTING IN THE TRADI- TIONAL MEANSHIFT ALGORITHM}

\subsection{Problem of Initialization}

A goal template contains moving target needs to be manually determined while the system is initialized [4]. The system error maybe big, This situation can't meet the requirements of intelligent tracking and accurate tracking.

\subsection{Problem of Kernel Function's Bandwidth}

while the system is in initial operation, first of all, a target area (tracking area) is determined in the first frame image. The width of the rectangular box is the bandwidth of kernel function [5], In the process of the whole algorithm runs, bandwidth is constant. When the shape or the area of the moving target area changes, the target tracking rectangular might be too big or too small. In the template and the candidate target template matching process, the algorithm takes the previous frame center of the target as a starting point for the current frame, and from this, begins to search the most similar target template following the direction of the probability density increasing. The range of Target can be correctly determined is within the scope of bandwidth of the kernel function, If the target moves too fast, the distance between the center of the last frame target and the center of the current frame target distance is too big, that is out of range, tracking will fail.

\subsection{Problem of the Color Distribution}

The traditional algorithm takes the color distribution characteristics in the target area (color histogram) as the judgment standard [6], only considers the overall characteristic of the color distribution without considering the location of the color information. when the algorithm determines the target template of rectangular area, part of the background image may be included., that is to say, the target template consists of background's color and goal's color. For getting the accurate target template, the Algorithm must make Meanshift vector moving in the direction of the target color increasing, and constantly iterating in order to obtain the most similar value. If the distance between background color and template center is greater than the distance between the target and template center, Or the decreasing rate of the background pixels is more than the decreasing rate of target template, Meanshift vector will be moving in the direction of the background pixels increasing, departs from the correct iteration direction, the algorithm cannot track the right goal.

\subsection{Experiment of Traditional Meanshift Algorithm}

Experiment Figs. (1-4) shows the effect of traditional Meanshift algorithm for target tracking under complex background. 


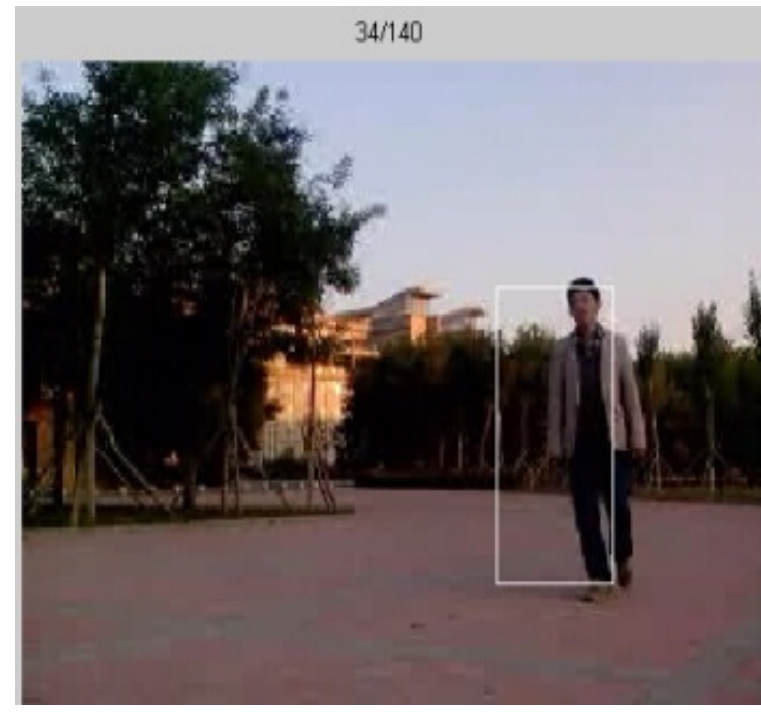

Fig. (1). The $34^{\text {th }}$ frame.

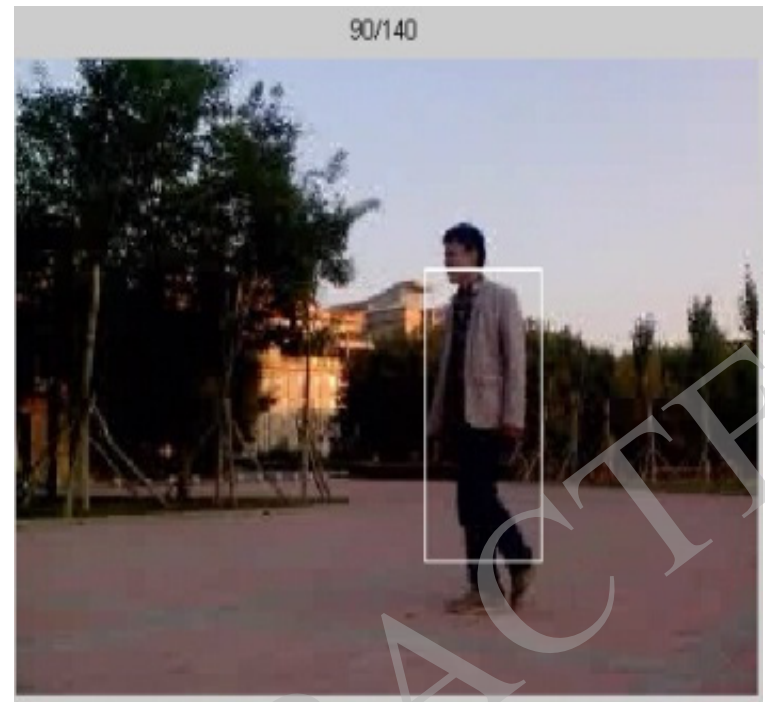

Fig. (2). The $90^{\text {th }}$ frame.

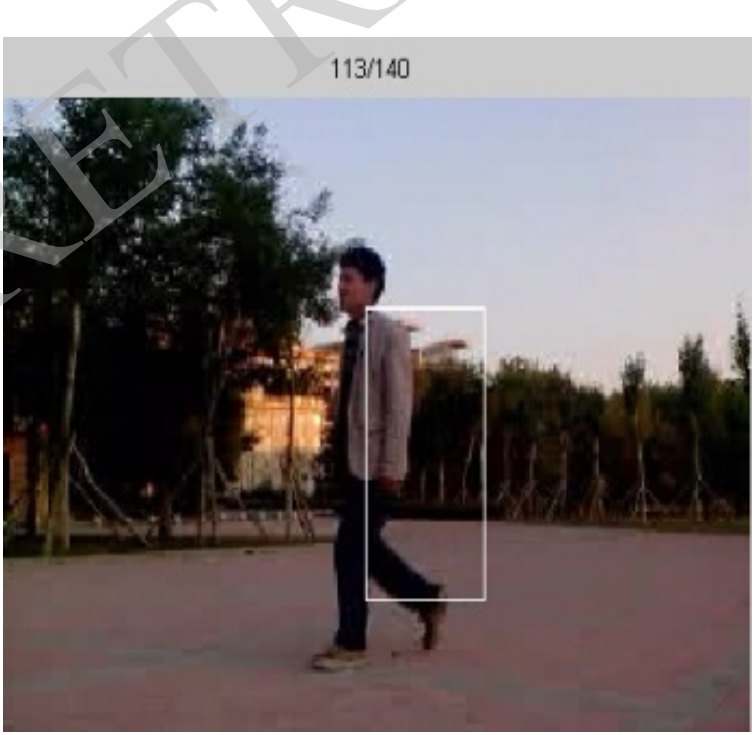

Fig. (3). The $113^{\text {th }}$ frame.

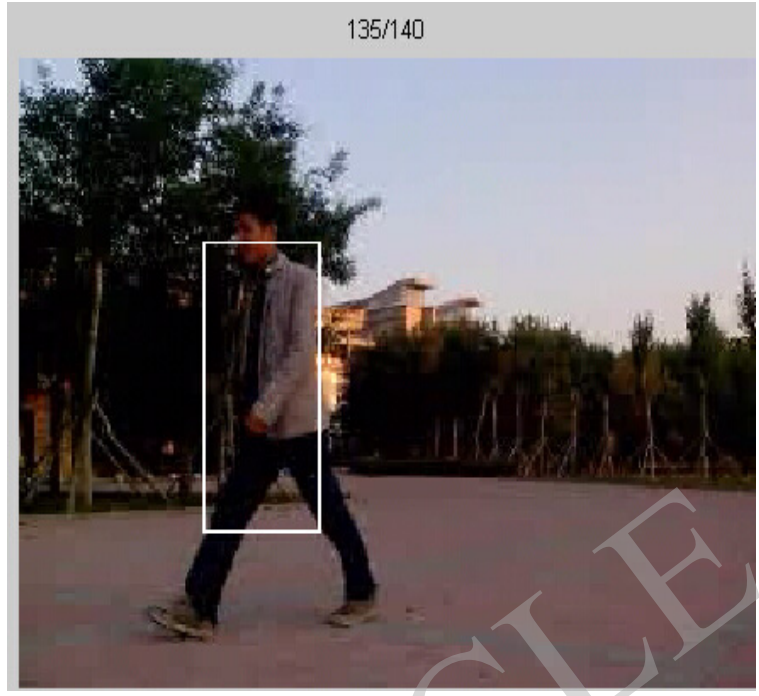

Fig. (4). The $135^{\text {th }}$ frame.

Figs. (1-4) are respectively the tracking effect of frame $34,90,113,135$ in image sequence, Meanshift algorithm basically completed the target tracking, while the tracking effect is not ideal, tracking migration phenomenon is relatively serious, there are obvious deviation in the 34th frame and the 113th frame. the target area in rectangular box is unstable. For the movement target tracking with complicated background, Meanshift algorithm is sensitive to the color, so the effect is not too good.

\section{AUTOMATIC INITIALIZATION OF MOVING TARGET}

In traditional Meanshift algorithm, manually locate the target area was located manually, and the errors will exist inevitably [7]. But Intelligent tracking a moving target requirements for automatic identification and access to moving targets. The trace rectangular was determined by detecting the binarization of the target area [8], and further the target centroidal position was determined, and the goal of the template initialization was achieved.

\subsection{The Determination of Mass's Center}

Assume that $\mathrm{B}(\mathrm{x}, \mathrm{y})$ is a moving target detection after binarization of the area, White areas represent moving target region and background region is black area, the entire movement target area was scanned and The coordinates of all pixels in the movement target area were remembered. The average of the abscissa of All pixel gray value within the target area is considered to be the center of mass [9] of the abscissa. The average of the ordinate of All pixel gray value within the target area is considered to be the vertical center of mass, assuming that the target centroidal coordinates is $(\mathrm{X}, \mathrm{Y})$ and $\mathrm{N}$ is the total number of the target area of pixels.

The $\mathrm{x}$ coordinate calculation formula:

$X=\frac{\sum_{m=1}^{N} X_{m}}{N}$

The y coordinate calculation formula: 
$Y=\frac{\sum_{m-1}^{N} Y_{m}}{N}$

\subsection{The Determination of the Destination Rectangle Box}

The binarization of the target area was searched in horizontal direction, the minimum horizontal notes for $\mathrm{x} 1, \mathrm{Ab}$ scissa largest recorded as $\mathrm{x} 2$. Similarly, in the vertical direction to the target area was searched, Minimum ordinate was noted for $\mathrm{y}_{1}$, the biggest ordinate was noted for $\mathrm{y}_{2}$,

The height of the rectangle:

$h=\frac{y_{2}-y_{1}}{2}$

The width of the rectangle:

$w=\frac{x_{2}-x_{1}}{2}$

\section{IDENTIFYING THE TARGET CENTER BASED ON PIXEL LOCATION}

For Traditional Meanshift algorithm, the color target model is established using the overall distribution characteristics of the target area, Only color statistical properties are considered, The location of the pixel features not be considered, the calculations are not enough accurate, Target color similar or complex are easy to lead to the failure tracking. The corresponding improving methods: improve the calculation method of the center of the candidate target template by Calculating the center of mass each color group in the target area. Pixel location information is embodied [10] by Calculating the centroidal position of each color group, Track will be more accurately and rapidly.

Each color unit (i) mass center:

$$
K_{i}^{n}=\frac{\sum_{i=1}^{n_{h}} \delta\left[b\left(X_{i}\right)-u\right] X_{i}}{\sum_{i=1}^{n_{h}} \delta\left[b\left(X_{i}\right)-u\right]}
$$

means $n$ frames, $\mathrm{Xi}$ means the position of pixels in the area of target, $u$ is histogram color values, $b\left(X_{i}\right)$ is the color value of pixels at $\mathrm{Xi}$.

By Calculating the center of mass of each color unit [11], Target ' $\mathrm{s}$ position in the center of the current frame [12] is obtained by calculating the mean each color unit. Calculation is as follows:

$$
y_{0}=\frac{\sum_{i=1}^{m} \alpha_{i} K_{i}^{n}}{\sum_{i=1}^{m} \alpha_{i}}
$$

$\alpha_{i}=T\left(\frac{b_{i}}{t_{i}}\right) t_{i}$

$\mathrm{T}$ (bi/ti) is weighting function, ti and bi respectively represent the A pixel's probability density [13] in the target region and background region. When bi/ti<E, $T(b i / t i)$ is 1 ; When bi/ti is other values, $\mathrm{T}(\mathrm{bi} / \mathrm{ti})$ is zero.
In the next frame, the candidate target centroidal position calculation formula:

$y_{1}=\frac{\sum_{i=1}^{m} \alpha_{i} K_{i}^{n+1}}{\sum_{i=1}^{m} \alpha_{i}}$

The improved MeanShift algorithm has the following advantages:

(1) Automatic tracking the target can avoid the error of the artificial participation and makes the tracking more accurately.

(2) Target pixel location information is embodied When calculating the candidate target centroidal point. Reduced the tracking deviation phenomenon [14] when target was matched by The traditional Meanshift algorithm with Color distribution as a whole.

(3) When calculating the candidate target center Traditional Meanshift algorithm needs many iteration times to get to the right place [9]. The improved algorithm gets the center of the next frame target after only once or several times. Computing speed is higher Significantly

\section{TARGET TRACKING PROCESS}

The target tracking process is shown in Fig. (5).

1). Using the moving target detection algorithm to obtain binary images of moving targets.

2). Area of the moving target are obtained by test, Determining the minimum circumscribed rectangle of moving targets, the bandwidth of the kernel function and the location of the initial target by The motion has been detected target area.

3). Locating the tracking target location and initializing the Meanshift algorithm by the tracking area which has been got.

4). Calculating the initial target centroid of each color group oft he initial goal by the formula (5).

5). Calculating the moving target centroid position in the initial frame by the formula (6), Calculating the next frame target by the formula.

6). Circling the steps above, finally realizes the tracking of moving targets.

\section{THE EXPERIMENTAL RESULTS AND ANALYSIS}

There are 2 experiments used the improved meanshift algorithm to track.

\subsection{Experiment 1}

The first experiment is shown in Figs. (6-9). This algorithm can automatically track the target and hardly offset for tracking the moving target. In the background and the target of similar cases, also can realize accurate tracking of the moving target. 

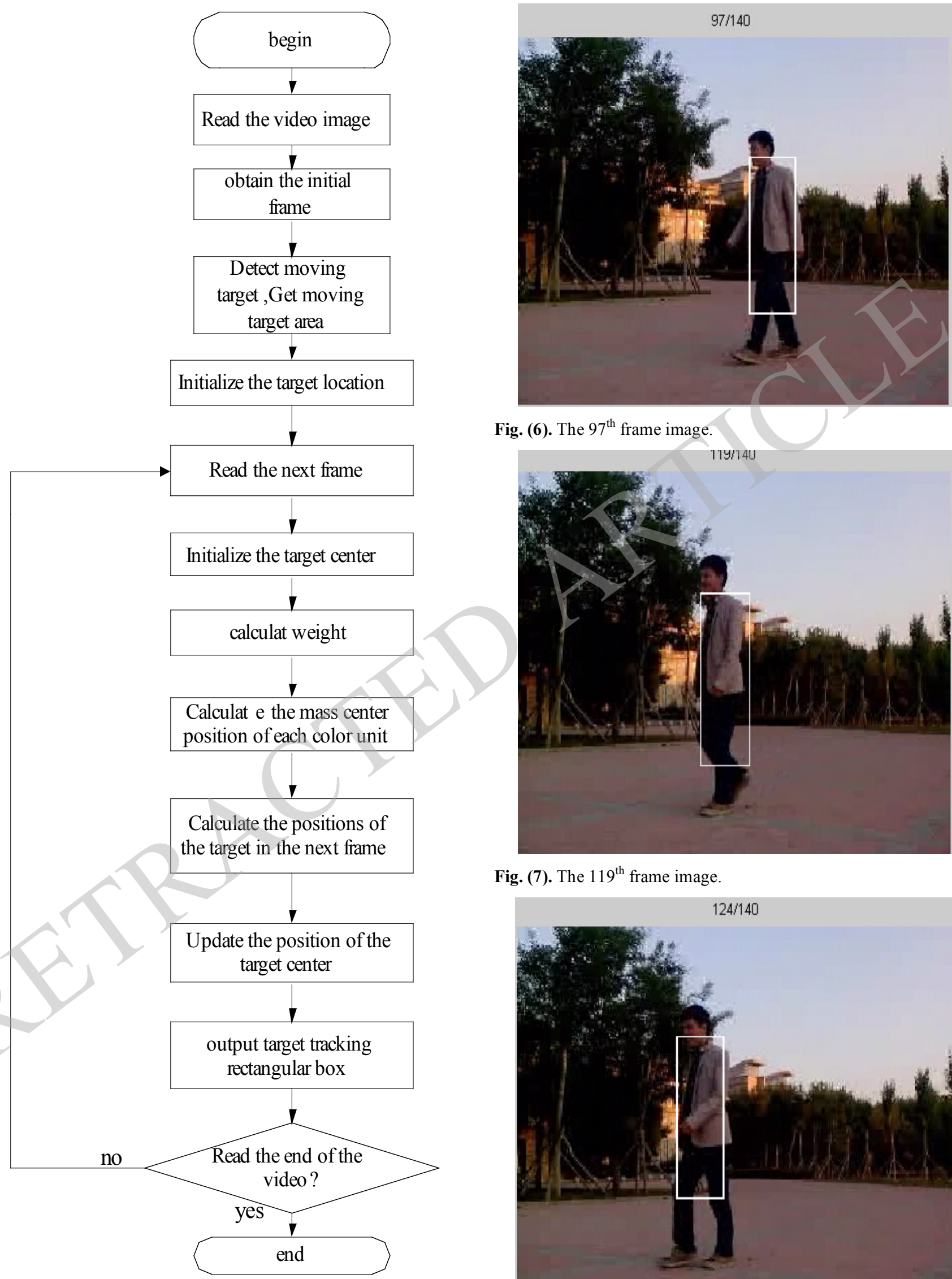

Fig. (6). The $97^{\text {th }}$ frame image.

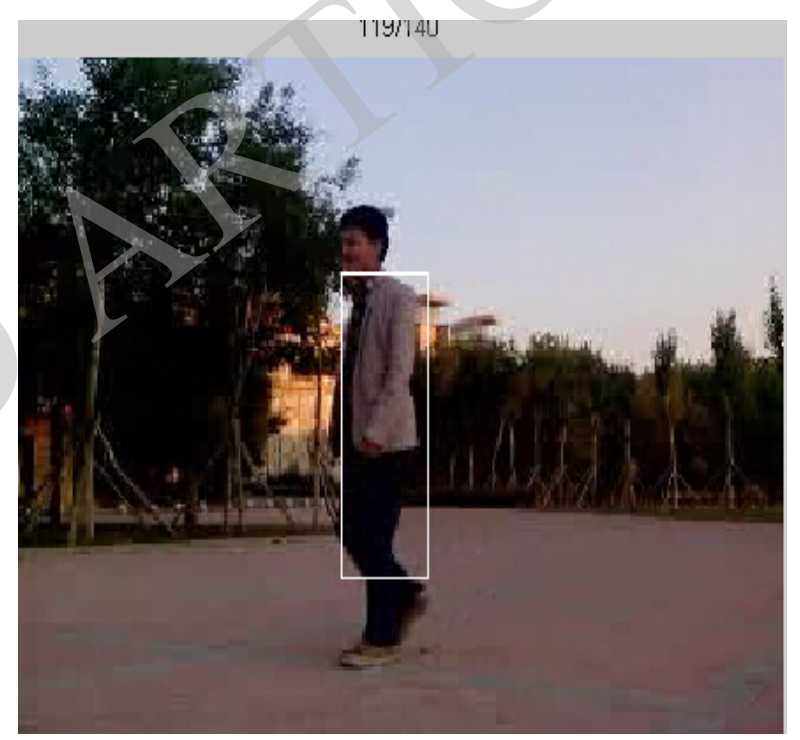

Fig. (7). The $119^{\text {th }}$ frame image.

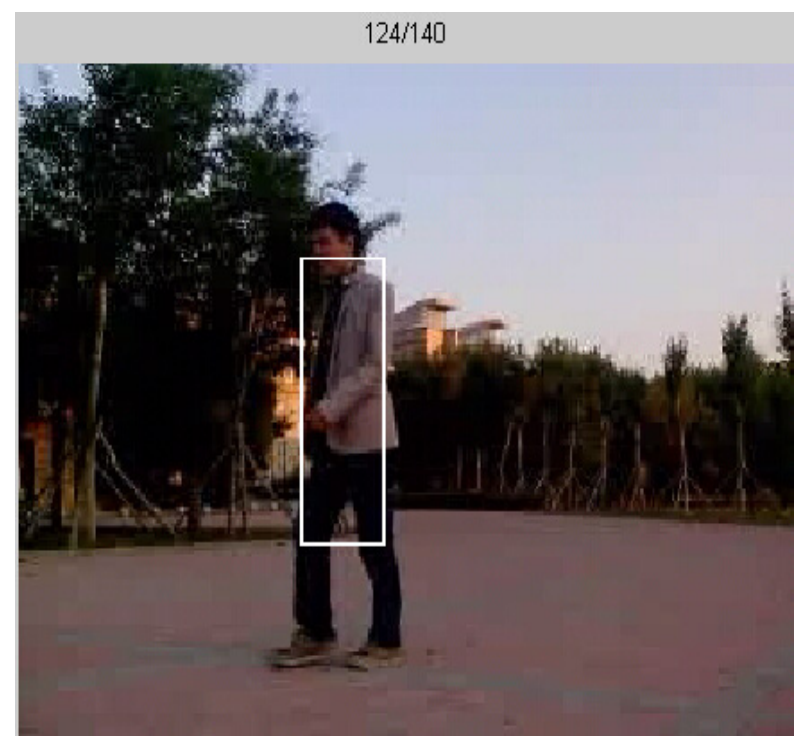

Fig. (5). The improved meanshift algorithm flow chart.

Fig. (8). The $124^{\text {th }}$ frame image. 


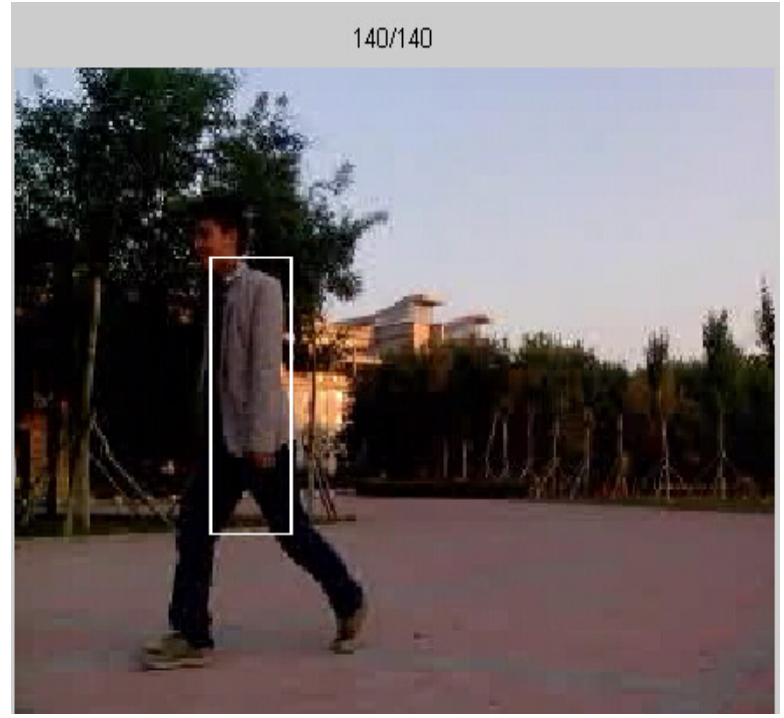

Fig. (9). The $140^{\text {th }}$ frame image.

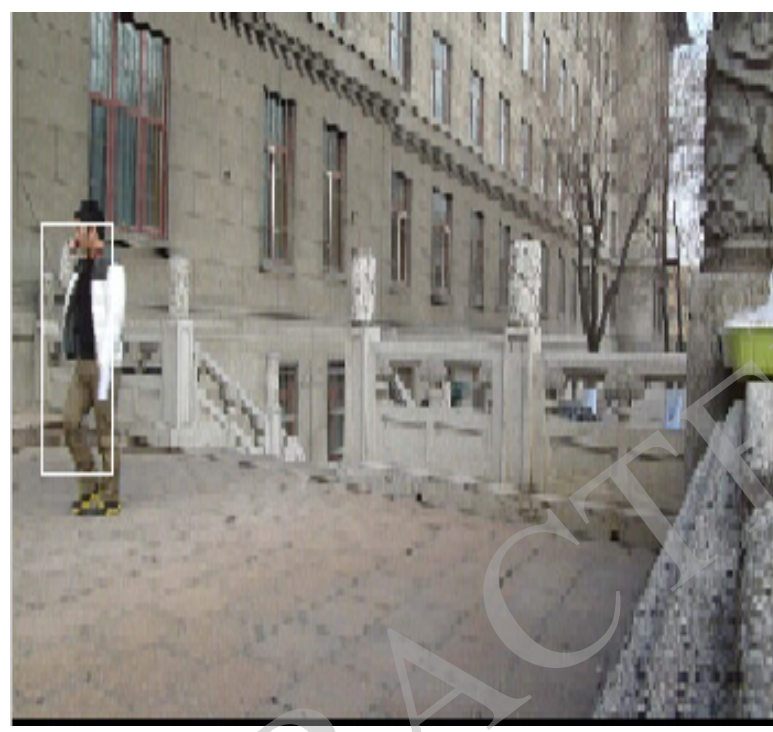

Fig. (10). The $34^{\text {th }}$ frame image.

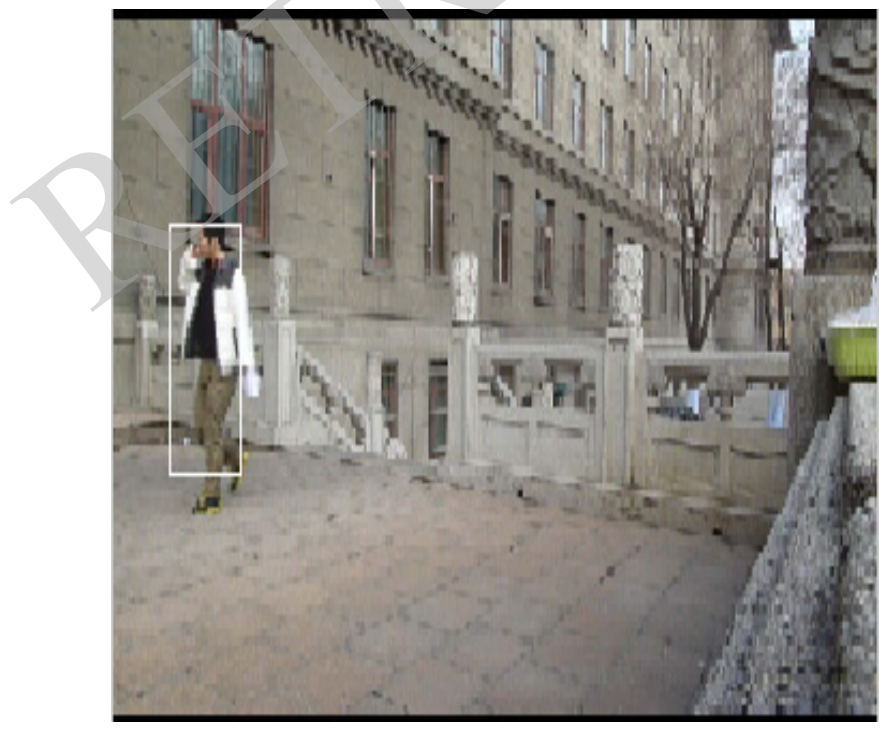

Fig. (11). The $48^{\text {th }}$ frame image.

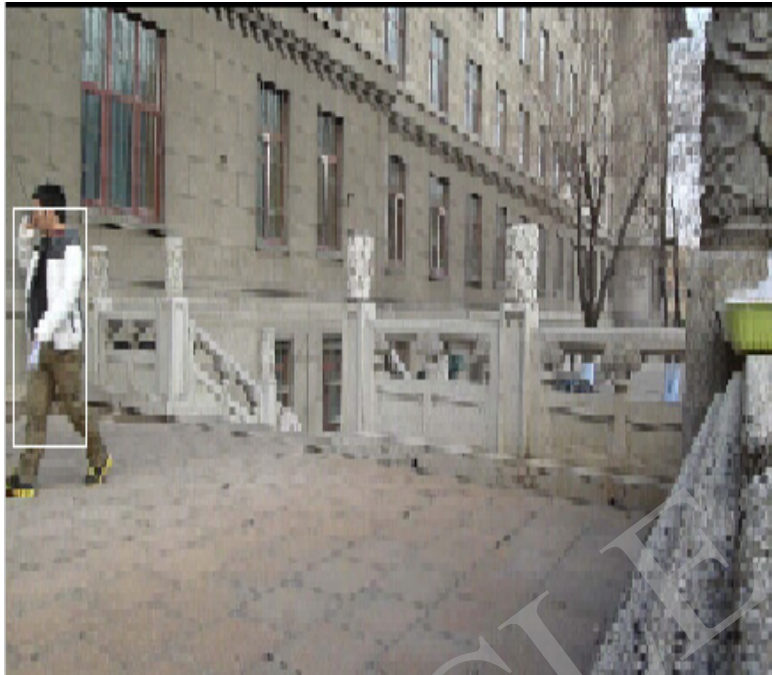

Fig. (12). The $64^{\text {th }}$ frame image.

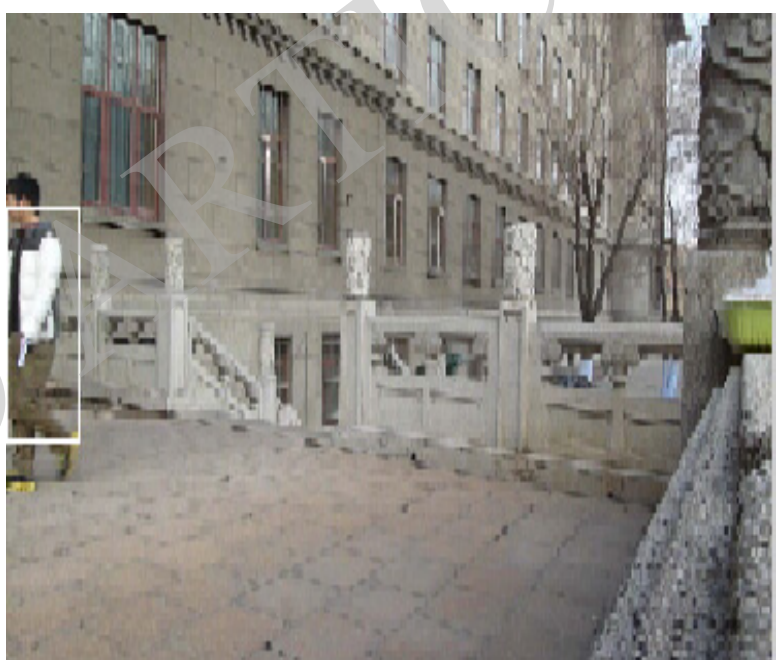

Fig. (13). The $72^{\text {th }}$ frame image.

\subsection{Experiment 2}

The second experiment is shown in Figs. (10-13). The experiment of the video background color is relative single, target and the background color have similar places, the algorithm can achieve the continuous tracking of target until the target is removed from the camera, the tracking is stably and accurately. The second experiment shows that the algorithm is good adaptability to the environment.

\section{CONCLUSION}

By improved Meanshift algorithm, the goal of the initialization is achieved automatically and centroidal position of each color group is determined, this improved meanshift algorithm avoids the error of the artificial participation, reduces the tracking deviation phenomena and the number of iterations when calculating the candidate target center. The tracking accuracy and efficiency are improved.

\section{CONFLICT OF INTEREST}

The authors confirm that this article content has no conflict of interest. 


\section{ACKNOWLEDGEMENTS}

This work is supported by the Industrial research Project of Liaoning Science and Technology Department, China (No.2012217005).

\section{REFERENCES}

[1] Chen Dong-yue, Chen Zong-wen, "Meanshift Robust Object Tracking Based on Feature Saliency", J. Journal of Shanghai Jiaotong University, 2013, 47(11), pp.1807-1812.

[2] Liu Qing, Tang Lin-bo, Zhao Bao-jun, Sun Jing-le, "Improved mean shift target tracking algorithm", J. Systems Engineering and Electronics, 2013, 35(6), pp.1318-1323.

[3] Dong Wen-hui, Chang Fa-liang, Li Tian-ping, "Adaptive Fragments-based Target Tracking Method Fusing Color Histogram and SIFT Features", J. Journal of Electronics \& Information Technology, 2013,35 (4), pp. 770-776.

[4] HUANG Zhen-wei,LI Xin-guo,"Study on Image Target Recognition and Tracking",J.Computer Simulation,2012,29(10),pp.283-287.

[5] DING Ye-bing,ZHAO Feng,HAO Shi-hai,"Multi-feature Bandwidth Adaptive Mean Shift Target Tracking Algorithm",J.Computer Engineering,2013,39(2),pp.197-201

[6] Liu Ming,Zhao Xiaolei, “Target tracking based on improved Camshift algorithm",J.Journal of Nanjing University of Science and Technology;2013,37(5),pp.755-760

[7] Cai Jia1, Huang Panfeng, "Research of a Real-time Feature Point Tracking Method Based on the Combination of Improved SURF and P-KLT Algorithm", J. Acta Aeronautica Et Astronautica Sinica, 2013, 34(5),pp. 1204-1214.

[8] Yang Min, Pei Ming-tao, Wang Yong-jie, Dong Zhen; WU Yu-wei, "Video-Based Vehicle Tracking Based on Moving Object Detection", J.Transactions of Beijing Institute of Technology, 2014 34(4),pp. 370-375.

[9] Xiu Chun-bo, Lu Shao-lei, Ren Xiao, "Improved Mean Shift tracking ajgorithm based on differentiaj informat", J.Systems Engineering and Electronics, 2014,36( 5),pp. 1004-1009.

[10] Zhang Zhi-long, Yang Wei-ping,Li Ji-cheng, “A Novel Salient Image Edge Detection Algorithm Based on Ant Colony Optimization", J. Journal of Electronics \& Information Technology, 2014,36 (9), pp.2061-2067.

[11] Jin Junfeng, Fang Qing, Tian Minghui, "Detection segment based airport target extraction", J.Journal of Computer Applications, 2013,33(S1), pp.310-314

[12] Zou Wei, Zhao Xunjie, Li Quan, Lu Kai, “A MULTI-TARGET PEDESTRIAN TRACKING ALGORITHM BASED ON MOTION DETECTION", J. Computer Applications and Software, 2014,31( 8), pp.132-135.

[13] Xu Yan, SHI Zai-feng, LI Bin-qiao, Pang Ke, "Neighborhood Calculation Histogram Equalization Enhancement Algorithm for Video Image", J.Computer Engineering, 2014, 40(10), 245-251.

[14] Yang Hui, Liu Jun, Ruan Song, "Study on video tracking based on Mean Shift algorithm", J. Computer Engineering and Design, 2013, 34(6), 2062-2066.

(C) Zhou et al.; Licensee Bentham Open.

This is an open access article licensed under the terms of the Creative Commons Attribution Non-Commercial License (http://creativecommons.org/licenses/by-nc/3.0/) which permits unrestricted, non-commercial use, distribution and reproduction in any medium, provided the work is properly cited. 